\title{
FLEXURAL PROPERTIES OF SYNTACTIC FOAM REINFORCED BY WARP KNITTED SPACER FABRIC
}

\author{
Chao Zhi ${ }^{1,2}$, Hairu Long ${ }^{1,2 *}$ \\ ${ }^{1}$ College of textiles, Donghua University, Shanghai 201620, China \\ ${ }^{2}$ Engineering Research Center of Technical Textiles, Ministry of Education, Shanghai 201620, China \\ *E-mail: hrlong@dhu.edu.cn
}

\begin{abstract}
:
The aim of this study was to investigate the flexural behaviours of syntactic foam reinforced by warp knitted spacer fabric (SF-WKSF). Seven kinds of SF-WKSF samples were fabricated by warp knitted spacer fabric (WKSF) with structural parameters including surface layer structures, inclination-angle and fineness of spacer yarns, different microballoons types and contents. The flexural tests were carried out and the bending properties of SF-WKSF were analysed based on the strength and modulus values obtained from the test results. It is indicated that the SF-WKSF shows higher flexural strength and modulus compared to neat syntactic foam with almost no impact on the density of composites. The results also demonstrate that the surface layer structure, inclination-angle of spacer yarns, microballons content and type have significant effects on the flexural responses of SF-WKSF. The composites exhibit better antibending capacities by selecting larger inclination-angle, closer surface layer structure, higher density and relatively higher content of S6OHS microballoons.
\end{abstract}

\section{Keywords:}

Syntactic foam, warp knitted spacer fabric, flexural properties

\section{Introduction}

Syntactic foams are defined as materials which are formed by embedding hollow microspheres - microballoons into a polymer matrix. The matrixes are usually thermosetting resins such as epoxy, polyester, phenolic, while the microballoons can be made from glass, phenolic, carbon and so on [1]. Syntactic foams exhibit excellent properties, that is, low weight, high specific strength and low moisture absorption. Because of this, these materials are commonly used in aerospace, marine, vehicles and architectural applications [2].

Flexural properties are important factors for the mechanical performance of syntactic foams. Many researchers have investigated the flexural properties of various syntactic foams [3-6]. Results of these researches showed that the addition of microballoons could reduce the densities of materials. The syntactic foams commonly have a higher specific flexural modulus and even a higher flexural modulus compared to the neat resin. Nevertheless, a higher volume fraction of microballoons leads to a lower flexural strength of syntactic foams resulting in the restriction of applications. Presently, embedding short fibres in syntactic foams is a viable means for enhancing the flexural properties of syntactic foams. Many researchers reported that the incorporation of chopped strand glass fibres or carbon fibres could improve the flexural properties of syntactic foams [1, 7-9]. However, this method has some limitations; short fibres are hard to uniform disperse and may cluster and bunch in the matrix. Due to the clustering and bunching, the fibres are not completely wet by the matrix hence the fibres are less effective in bearing the load. And even worse, the incomplete wet fibres can make the interface region to be filled with air and eventually reduce the mechanical properties of the whole materials $[7,10]$.

WKSF has much better cushion properties. The published studies conducted on WKSF or composites enhanced by WKSF are mainly the focus on their compressive and impact properties [11-13]. In these researches, WKSF showed good cushioning performance, and the great structural integrity of WKSF could prevent the clustering and bunching of reinforced fibres. Therefore, WKSF is especially adapted for the use of skeleton materials in composites.

In this study, syntactic foam reinforced by WKSF (SF-WKSF) was fabricated and the flexural properties of SF-WKSF were compared with NSF. Meanwhile the effects of structural parameters of WKSF, microballoons content and type on the flexural performance of SF-WKSF were also investigated and discussed. In addition, the fracture features of SF-WKSF and NSF under flexural load were done through macro-observation and scanning electron microscope (SEM).

\section{Experimental}

\subsection{Materials}

A kind of ambient-temperature curing epoxy resin system consisting of diglycidyl ether of bisphenol-A epoxy resin (E-51) 
and amine epoxy hardener (100-1B) (Wuxi Singmen Electronic Materials Co. Ltd, China) were used for the matrix materials of syntactic foams. Two types of hollow glass microballoons (3M Co., MN) with trade name of $\mathrm{K} 1$ and $\mathrm{S} 60 \mathrm{HS}$ were used as the light-weight filler. The physical properties of these microballoons are listed in Table 1, in which the true density, mean particle size and strength are provided by the supplier, and the radius ratio $(\eta)$ was calculated using the following equation:

$$
\eta=\left(1-\frac{\rho_{m}}{\rho_{g}}\right)^{\frac{1}{3}}
$$

where $\rho m$ is the density of microballoons, and the value is obtained from the manufacturer's datasheet; $\rho g$ is the density of glass, in this work the value is $2.50 \mathrm{~g} / \mathrm{cm}^{3}$. The higher $\eta$ value will result in the thinner microballoons' wall.

WKSF was produced on double-needle-bar Raschel warp knitting machine of E-18 (Wuyang Co., Ltd, China). The working principle of this machine is shown in Figure 1 [14]. The guide bars GB1 and GB2 knitted a surface layer of WKSF on the front needle bar, while the guide bars GB5 and GB6 made another surface layer of WKSF on the back needle bar. The guide bars GB3 and GB4 carried the spacer yarns and looped alternately both on the front and back needle bars to connect two surface layers.

Four types of WKSF namely B1, B2, B3 and B4 with different structural parameters were involved in this work. All WKSF contain two types of inclination-angle of spacer yarns (characterized by lapping code), surface layer structures and fineness of spacer yarns. Polyester monofilament was applied for spacer yarns and polyester multifilament (DTY) of 300D/96F for surface layer yarns. The detailed parameters of spacer yarns and WKSF are listed in Tables 2 and 3, respectively. Figure 2 presents the structural features of WKSF.

\subsection{Sample preparation}

To disperse the microballoons uniformly, the temperature of the epoxy resin remained $60 \square$ during the mixing process. A weighed quantity of microballoons was added to the epoxy resin for several times, and each time half of the remaining

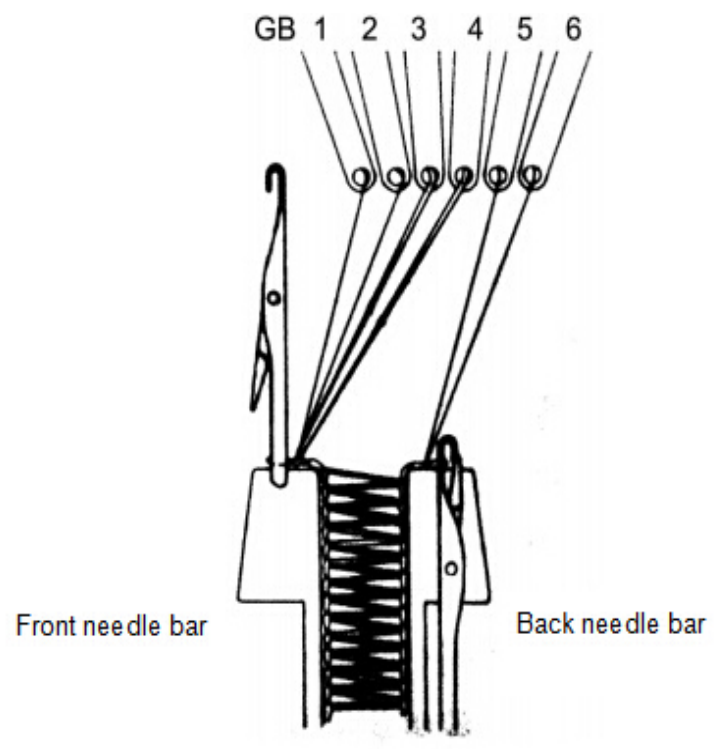

Figure 1. Working principle of double-needle-bar warp knitting machine

Table 1. Specifications of microballoons used in fabricating syntactic foam specimens

\begin{tabular}{|c|c|c|c|c|}
\hline $\begin{array}{c}\text { Microballoon } \\
\text { type }\end{array}$ & $\begin{array}{c}\text { True density } \\
(\mathbf{g} / \mathbf{c m} \mathbf{)}\end{array}$ & $\begin{array}{c}\text { Mean particle size } \\
(\boldsymbol{\mu m})\end{array}$ & Radius ratio & $\begin{array}{c}\text { Pressure for 90\% survival } \\
(\mathbf{M P a})\end{array}$ \\
\hline K1 & 0.125 & 65 & 0.98 & 1.72 \\
\hline S60HS & 0.600 & 30 & 0.91 & 124.02 \\
\hline
\end{tabular}

Table 2. Technology parameters of spacer yarns

\begin{tabular}{|c|c|l|}
\hline Type & $\begin{array}{c}\text { Diameter } \\
(\mathbf{m m})\end{array}$ & \multicolumn{1}{c|}{ Lapping code, Threading } \\
\hline A & 0.2 & $\begin{array}{l}\text { GB3:1-0 3-2/3-2 1-0//, 1 full 1 empty } \\
\text { GB4:3-2 1-0/1-0 3-2//, 1 empty } 1 \text { full }\end{array}$ \\
\hline B & 0.2 & $\begin{array}{l}\text { GB3:1-0 4-3/4-3 1-0//, 1 full } 1 \text { empty } \\
\text { GB4:4-3 1-0/1-0 4-3//, 1 empty } 1 \text { full }\end{array}$ \\
\hline C & 0.16 & $\begin{array}{l}\text { GB3:1-0 4-3/4-3 1-0//, 1 full 1 empty } \\
\text { GB4:4-3 1-0/1-0 4-3//, 1 empty } 1 \text { full }\end{array}$ \\
\hline
\end{tabular}

Table 3. Structural parameters of WKSF

\begin{tabular}{|c|c|c|c|c|c|c|c|}
\hline No. & Texture of surface layer & $\begin{array}{c}\text { Thickness } \\
\mathbf{( m m})\end{array}$ & $\begin{array}{c}\text { Area } \\
\text { density } \\
\mathbf{( g / m 2 )}\end{array}$ & $\begin{array}{c}\text { Course } \\
\text { density } \\
\text { (wales/5cm) }\end{array}$ & $\begin{array}{c}\text { Wale } \\
\text { density } \\
\text { (courses/5cm) }\end{array}$ & $\begin{array}{c}\text { Spacer } \\
\text { yarn } \\
\text { type }\end{array}$ & $\begin{array}{c}\text { Spacer yarn } \\
\text { distribution } \\
\text { density } \\
\text { (number/cm2) }\end{array}$ \\
\hline B1 & $\begin{array}{c}\text { Chain+ } \\
\text { Inlay }\end{array}$ & 7.588 & 900.86 & 35.150 & 27.100 & $\mathrm{~A}$ & 57.14 \\
\hline B2 & $\begin{array}{c}\text { Chain+ } \\
\text { Inlay }\end{array}$ & 7.640 & 910.35 & 35.650 & 27.350 & $\mathrm{~B}$ & 38.97 \\
\hline B3 & Hexagon-al mesh & 7.616 & 756.21 & 35.375 & 28.950 & $\mathrm{~A}$ & 30.71 \\
\hline B4 & $\begin{array}{c}\text { Chain+ } \\
\text { Inlay }\end{array}$ & 7.566 & 860.68 & 34.175 & 28.200 & $\mathrm{C}$ & 39.52 \\
\hline
\end{tabular}




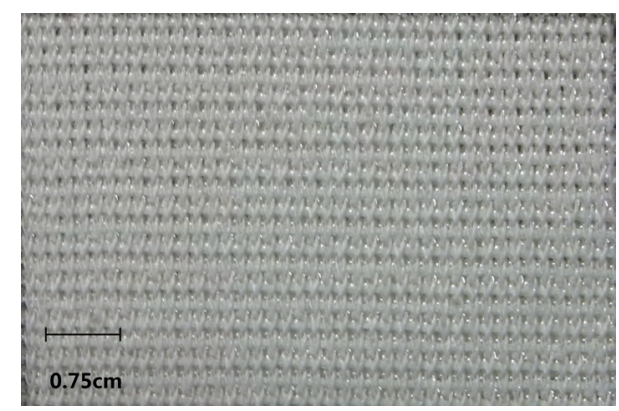

(a)

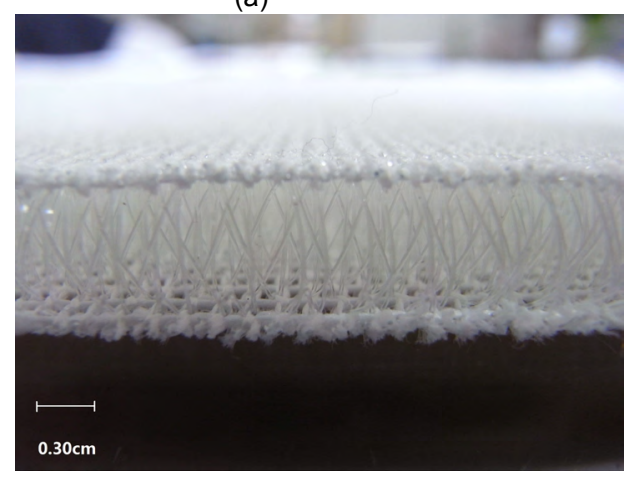

(c)

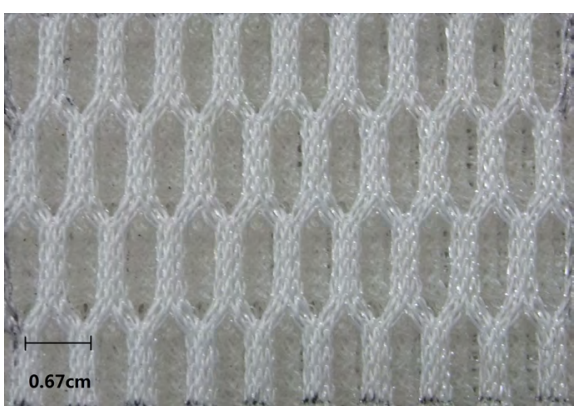

(b)

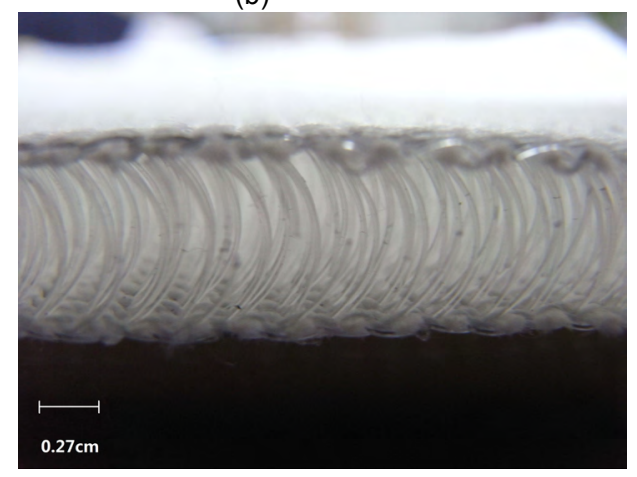

(d)

Figure 2. The structure of WKSF: (a) chain+inlay, (b) hexagonal mesh, (c) front view of spacer yarns and (d) lateral view of spacer yarns

microballoons was added. Then, the hardener was slowly added to the suspension. The mass ratio of resin and hardener were sustained at 100:33. The whole mixing process was done gently by using a wooden stirrer in order to avoid the breakage of microballoons. The slurry was filled into a cylindrical silicon mould with the size of $80 \mathrm{~mm} \times 10 \mathrm{~mm} \times 8 \mathrm{~mm}$. The WKSF, cut into $80 \mathrm{~mm}$ in length and $10 \mathrm{~mm}$ in width, was carefully pressed into the mixture. Then the specimens were cured $24 \mathrm{~h}$ at room temperature and postcured at $60 \square$ for additional $2 \mathrm{~h}$. Figure 3 represents the schematic illustration of SF-WKSF specimen. For comparison, the NSF specimen was prepared, and the specifications of all samples studied in this work were summarized in Table 4 . The densities of specimens were obtained according to the Archimedes principle. At least five specimens were used to measure the density. It is noted that the measured density of samples is lower compared to the theoretical density. It is due to the fact that the presence of void in the composite system. The void volume fractions $(V v)$ were calculated by using measured and theoretical densities as given by equation (2) [15].

$$
V_{v}=\frac{\rho_{t}-\rho_{m}}{\rho_{t}}
$$

where $\rho t$ and $\rho m$ are the theoretical density and measured density of specimens, respectively. The $\rho t$ was calculated based on the volume fraction of the various constituents and shown in equation (3).

$$
\rho_{t}=\rho_{t 1} \times V_{\% 1}+\rho_{t 2} \times V_{\% 2}+\rho_{t 3} \times V_{\% 3}+\ldots
$$

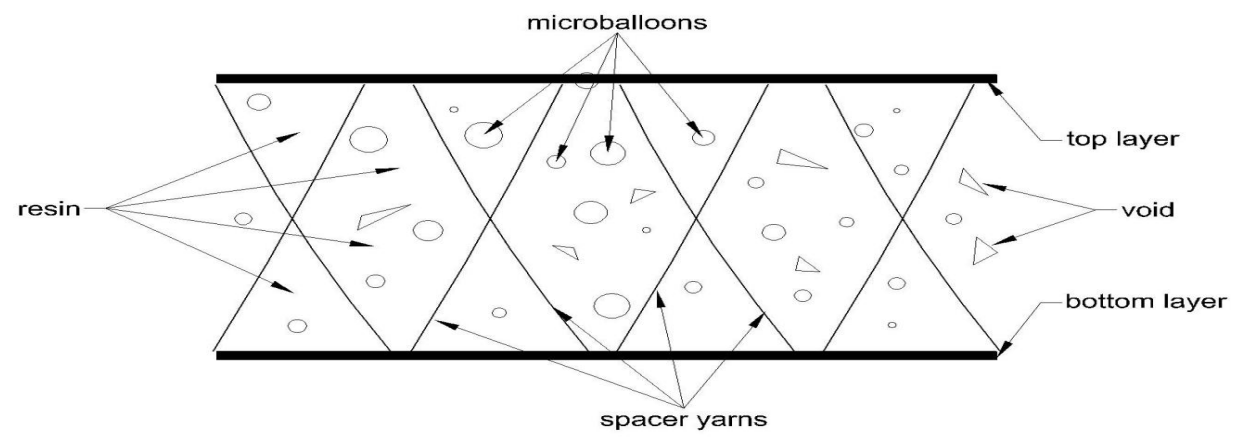

Figure 3. Schematic illustration of SF--WKSF 
Table 4. Sample specifications

\begin{tabular}{|c|c|c|c|c|c|c|}
\hline Samples & $\begin{array}{c}\text { Microballoon } \\
\text { type }\end{array}$ & $\begin{array}{c}\text { Microballoon } \\
\text { volume per } \\
\mathbf{c e n t} \\
\mathbf{( \% )}\end{array}$ & $\begin{array}{c}\text { WKSF } \\
\text { type }\end{array}$ & $\begin{array}{c}\text { Theoretical } \\
\text { Density } \\
\text { (g/cm3) }\end{array}$ & $\begin{array}{c}\text { Measured } \\
\text { Density } \\
\text { (g/cm3) }\end{array}$ & $\begin{array}{c}\text { Void volume } \\
\text { fraction } \\
\mathbf{( \% )}\end{array}$ \\
\hline S1 & S60HS & 30 & B1 & 1.024 & 0.971 & 5.17 \\
\hline S2 & S60HS & 30 & B2 & 1.024 & 0.969 & 5.37 \\
\hline S3 & S60HS & 30 & B3 & 1.019 & 0.945 & 7.26 \\
\hline S4 & S60HS & 30 & B4 & 1.022 & 0.965 & 5.58 \\
\hline S5 & S60HS & 20 & B1 & 1.075 & 1.030 & 4.19 \\
\hline S6 & S60HS & 40 & B1 & 0.972 & 0.905 & 6.89 \\
\hline S7 & K1 & 30 & B1 & 0.893 & 0.855 & 4.26 \\
\hline NSF & S60HS & 30 & - & 0.992 & 0.960 & 4.13 \\
\hline
\end{tabular}

where $\rho t 1, \rho t 2, \rho t 3 \ldots$ are the densities of different components and $V \% 1, V \% 2, V \% 3 \ldots$ are the correspondent volume fraction of these components in the whole composite system. The densities of neat epoxy resin system and fibre materials (polyester) used in this research are $1.160 \mathrm{~g} / \mathrm{cm}^{3}$ and $1.380 \mathrm{~g} /$ $\mathrm{cm}^{3}$, respectively.

\subsection{Flexural test}

The ISO 14125:1998 standards are adopted for the three-point bending (TPB) tests. The TPB tests were performed with a span (L) of $64 \mathrm{~mm}$ and all specimens have a specimen length (I), width (w) and thickness ( $\mathrm{t}$ ) of $80 \mathrm{~mm}, 10 \mathrm{~mm}$ and $8 \mathrm{~mm}$, respectively. The schematic diagram of TPB texts is shown in Figure 4.

The TPB tests were carried out using HuaLong WDW-20 Electronic Universal Tester. The experimental instrument and process of flexural tests are shown in Figure 5. The constant deformation rate was $2 \mathrm{~mm} / \mathrm{min}$ and at least five specimens were tested. The tests lasted until the NSF specimens' fracture and the deflection (displacement) of SF-WKSF reached $6 \mathrm{~mm}$. Load and displacement data obtained from the tests were used to plot the load-displacement curves. Flexural strength $(\sigma)$ and flexural modulus (Ef) were calculated using equation (4) and (5), respectively.

$$
\sigma=\frac{3 P L}{2 w t^{2}}
$$

where $P$ is the peak load, $L$ is the span length, $w$ and $t$ are the width and thickness of the specimens, respectively.

$$
E_{f}=\frac{L^{3} K}{4 w t^{3}}
$$

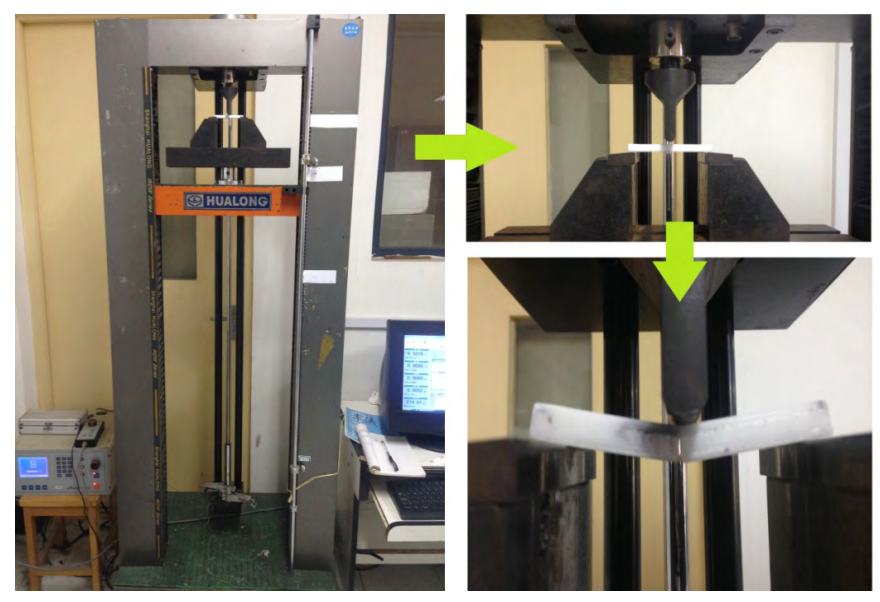

Figure 5. The experimental instrument and process of TPB tests

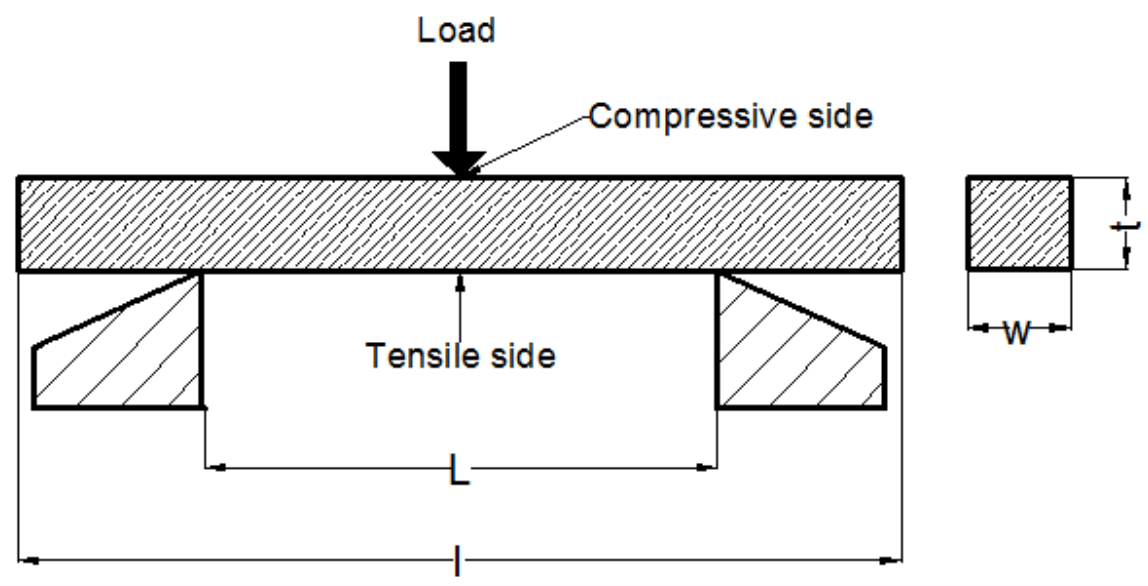

Figure 4. The test specimen configuration and schematic diagram of TPB texts 
where $K$ is the stiffness or the slope of the load-displacement curve in the initial portion.

\section{Results and discussions}

\subsection{Flexural properties of SF-WKSF and neat syntactic foam}

The TPB tests present a case where the load changes through the thickness direction of specimens. The load changes from compression at the compressive side to tension on the opposite surface of the specimens marked as tensile side (Figure 4). In addition, shear loads spread along the length of the specimens. In general, the specimens can fracture under two types of loads, horizontal load and vertical load. Hence, crack location and propagation directions can give an evaluation in determining the type of load that causes specimen failure [16]. It was observed that in all specimens, the failure process can be observed in the tensile side firstly and then followed by a sudden (NSF) or slow (SF-WKSF) failure. Figure 6 shows the front view of the specimens after TPB tests. It is noticed that cracks propagate in a curve manner for the NSF specimen, and the same occurs at an approximate oblique line manner for the SF-WKSF specimens. This phenomenon illustrates that the NSF specimen mainly bears the horizontal load along the length of specimens and SF-WKSF specimens are mainly imposed by the vertically load. The reason of the difference is that these cracks propagated at a random direction to the load based on the least resistance path, which results in the fact that the presence of WKSF especially the spacer yarns stops the transmission of cracks propagating along the length of the specimens and assist the straightening of the crack path.

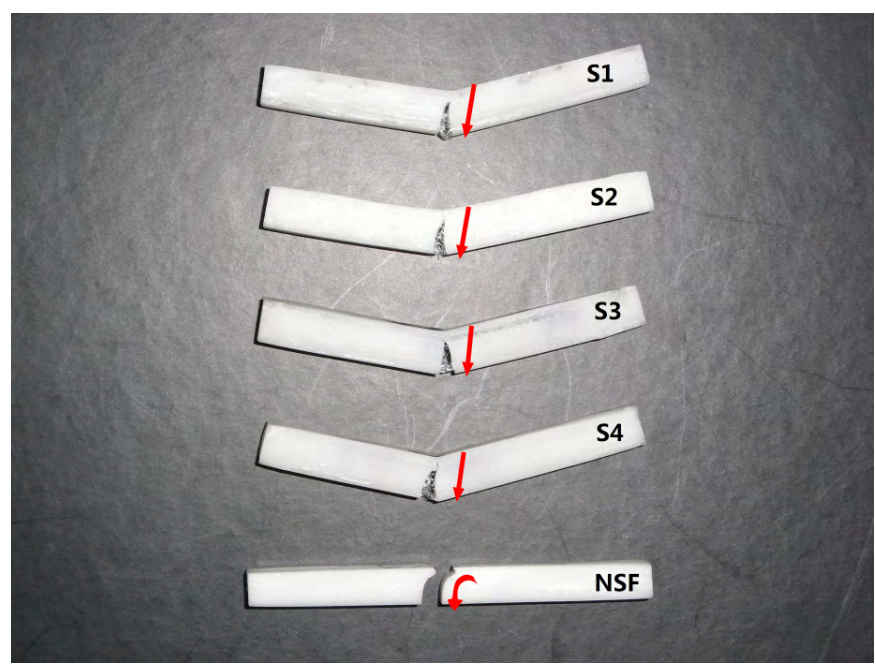

Figure 6. The front view of the specimens after TPB tests

Figure 7 displays the flexural load-displacement curves of SF-WKSF and NSF in TFB tests. Obviously, all of the curves display the similar linear trend in the initial phase and the load values decrease sharply after the end of the elastic region due to failure initiation in the specimens. The specimens of NSF show complete fracture, whereas the specimens of SF-WKSF show an approximate plateau region after this sharp decrease in the load. All the SF-WKSF specimens have a higher peak load than NSF composites. In addition, due to the introduction of surface layers, compared with NSF, all the samples of SFWKSF have a certain extent of strength after the sudden drop of load values, which is very important in engineering.

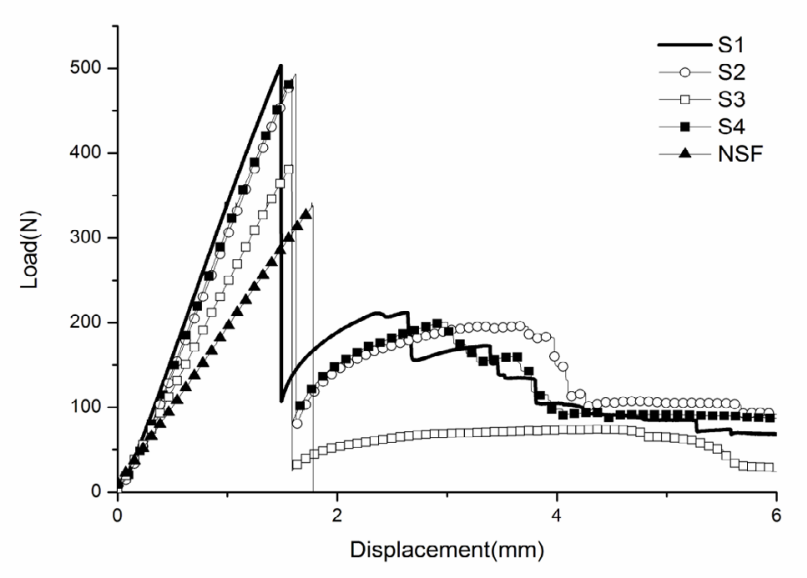

Figure 7. TPB test results of SF--WKSF and neat syntactic foam.

Figure 8 shows the flexural strength and flexural modulus of SF-WKSF and NSF. It can be seen from the bar chart that S1, S2, S3 and S4 have $49 \%, 46 \%, 14 \%$ and $44 \%$ higher values in flexural strength and $60 \%, 39 \%, 20 \%$ and $43 \%$ higher values in flexural modulus compared with NSF, respectively. In addition, the densities of S1, S2, S3, S4 and NSF are close to each other (Table 4). It can be concluded that the addition of WKSF improves the flexural properties of syntactic foams with almost no impact on the density of composites. According to the least resistance path theory as mentioned above, these cracks that produce through the TPB tests have the tendency to propagate along the least resistance direction, so the cracks of NSF samples have the curve manner as shown in Figure 6. When the WKSF is introduced into the syntactic foam, the presence of spacer yarns prevents the horizontal propagation of crack and changes the propagate paths of specimens from curve line to oblique line (Figure 6), and thus forces the cracks away from the least resistance path and increases the flexural properties of materials. In addition, it is obvious from the Figure 6 that a clear split of the NSF samples has occurred. In the case of SF-WKSF, the specimens do not readily split, and instead only a bent appearance can be seen for all the SF-WKSF specimens. This is primarily due to the presence of surface fabrics, which results in the reinforced foam taking more load than the unreinforced materials. Based on the two points, the introduction of spacer yarns and surface fabric are the reasons why SF-WKSF has better flexural strength and modulus compared with NSF.

The fracture surfaces of specimens were examined by SEM. Figure 9 shows the fracture details of NSF. It can be clearly seen that the step structure prevails for the microstructures of NSF. According to the least resistance path theory, it is assumed that when the NSF specimens were under flexural load, the cracks appeared and propagated through matrix resin. When they reach the interface between microballoons and matrix resin, cracks will be resisted and break away the microballoons due to the high-strength $\mathrm{S} 60 \mathrm{HS}$ microballoons. 
(a)

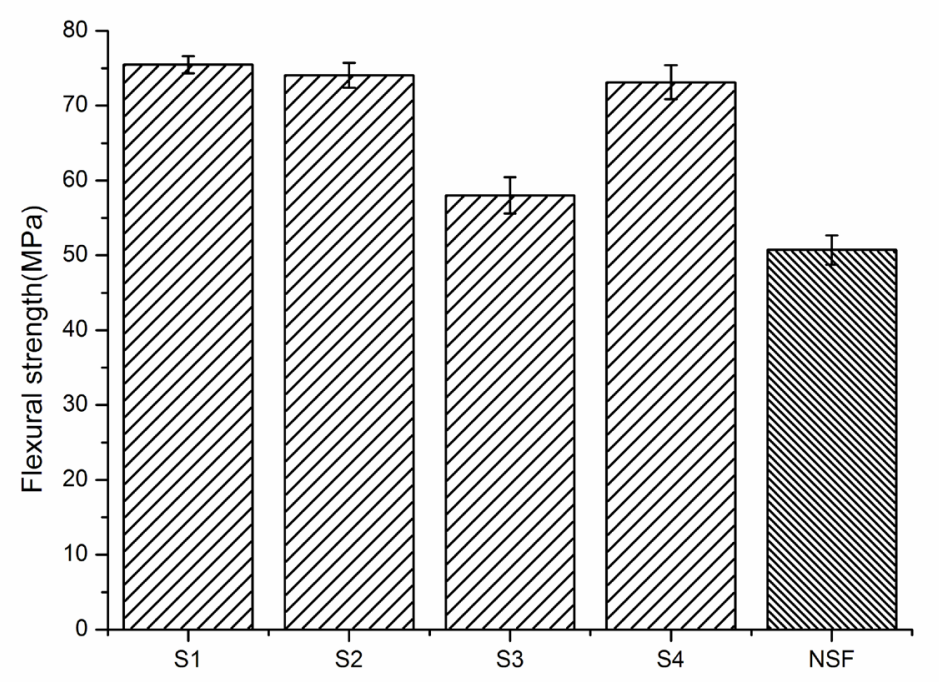

(b)

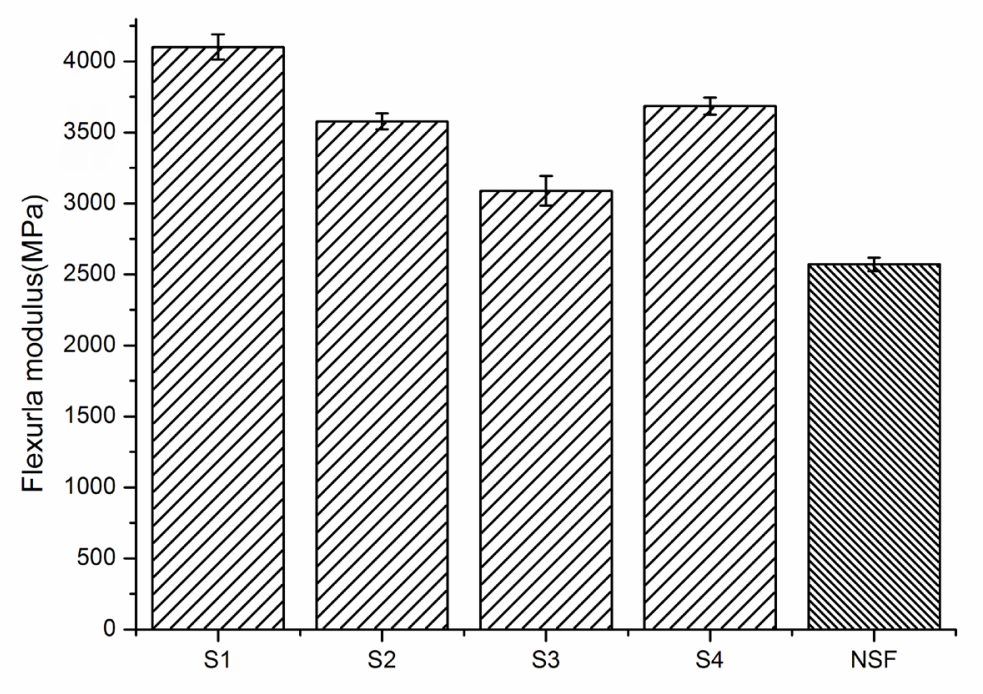

Figure 8. The (a) flexural strength and (b) flexural modulus of SF-WKSF and NSF

It can be proved that the fracture surface of NSF involves a small amount microballoon debonding and fracture, almost with no instance of the crushing of microballoons, as shown in Figure 9. Meanwhile, there are more step structures around microballoons compared with the other regions. This illustrates that the microballoons embedded in NSF can change the direction of crack. The microballoons are not the primary load carrier under TPB tests and the failure of NSF is dominated by resin matrix fracture. The presence of microballoons makes the cracks propagate at different planes and form more step structures. Furthermore, the step structures are considered as new surfaces, leading to more fracture energy-absorbed on them.

Figure 10 shows the SEM micrograph of fracture surface of specimen S1 after TPB tests. It is obvious that the step structures can be found in SF-WKSF. And the spacer yarns in SF-WKSF are pulled out and deformed during the TPB tests, indicating that the spacer yarns are one of the primary load carriers. The phenomena result in more irregular cracks which are considered as additional mini-type step structures absorbing more flexural energy. Furthermore, there are more step structures found around microballoons. And there is nearly no crushed microballoons that can be revealed during the TPB tests, as shown in Figure 10. Therefore, a conclusion could be drawn that the spacer yarns and matrix resin are the primary load carrier in SF-WKSF during the TPB process.

\subsection{Effects of WKSF on flexural properties}

Specimens S1 and S2 have the same structural parameters except the spacer yarns' inclination-angle of WKSF which depends on the lapping codes of guide bars GB3 and GB4. Figure 11 shows the global lapping movements of spacer yarns carried by GB3 for type I (red lines) and II (green lines). In the figure, the $x-, y$ - and $z$-axes indicate the direction of weft, wale, and thickness of spacer fabric, respectively. All the dots represent the junctions between spacer yarns and surface 


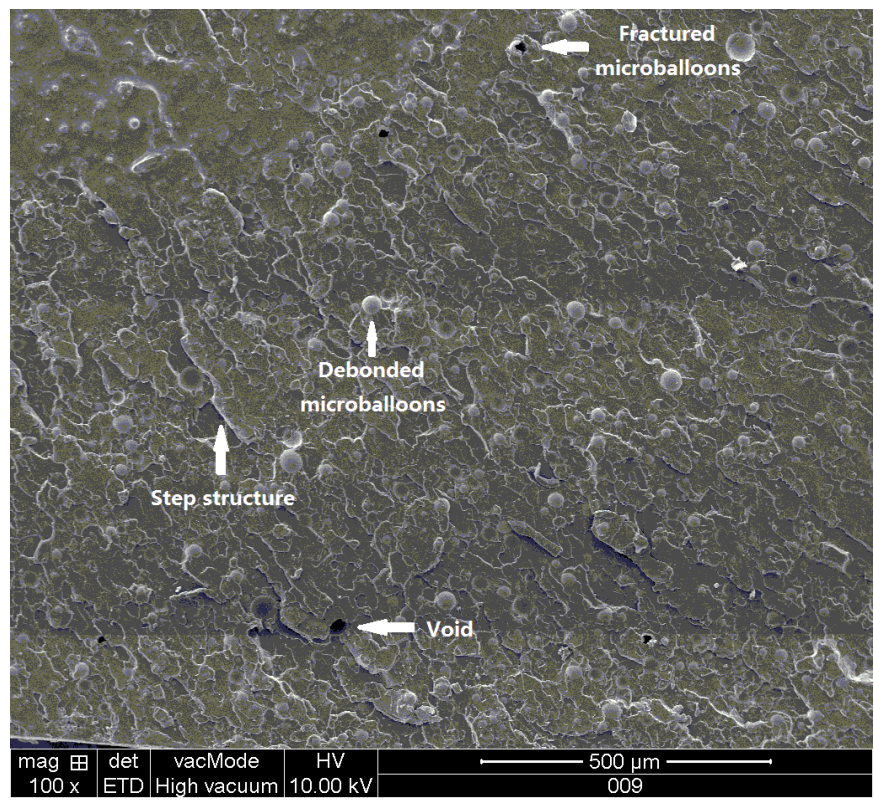

Figure 9. SEM micrograph of the fracture surface of NSF after TPB tests

layers. $\alpha$ and $\beta$ denote the spacer yarns' inclination-angles of WKSF B1 and B2, respectively. It is clear that the inclinationangle of spacer yarns for B1 $(\alpha)$ is larger than that of B2 $(\beta)$. Based on the flexural strength and modulus of $\mathrm{S} 1$ and $\mathrm{S} 2$ shown in Figure 8, it is evident that the sample with larger inclinationangle presents slightly higher flexural strength and $15 \%$ higher modulus value compared to the corresponding sample. The larger inclination-angle of spacer yarns $(\alpha)$, the shorter the length of spacer yarns $\left(A A^{\prime}\right)$ as a result of better critical force of spacer yarns. This increases the bending resistance abilities of SF-WKSF.

The spacer yarns with different diameters $(0.2 \mathrm{~mm}$ and 0.16 $\mathrm{mm}$ ) were used in specimens S2 and S4. The flexural loaddisplacement curves of S2 and S4 are fairly the same in the entire testing process. Similar tendency can be found in Figure 8 where the flexural strength and modulus values of S2 and S4 are close. The findings obtained demonstrate that the effect of fineness of spacer yarns on anti-bending capacities is not significant. This can be explained by the fracture mechanism of SF-WKSF which explains that the spacer yarns and matrix resin are the primary load carrier of SF-WKSF during the TPB process and the major failure mode of spacer yarns are pulled out and deformed without fracture. Thus, when the diameter of spacer yarns reaches a certain value to make the strength of spacer yarns reach a fairly high level, the fineness of the spacer yarn is not a critical factor on the flexural capacities of SF-WKSF.

The surface layer structures of WKSF B1 and B3 are Chain+Inlay and Hexagonal mesh, respectively. As shown in Figure 2, B1 presents much closer surface layer structures than that of B3. This illustrates that B1 has a higher area density and spacer yarns distribution density than B3 (Table 3). Referring to Figure 8 , it can be found that $\mathrm{S} 1$ reinforced with $\mathrm{B} 1$ has a significantly higher flexural strength and modulus compared with S3 conducted of B3. Closer surface layer structure leads

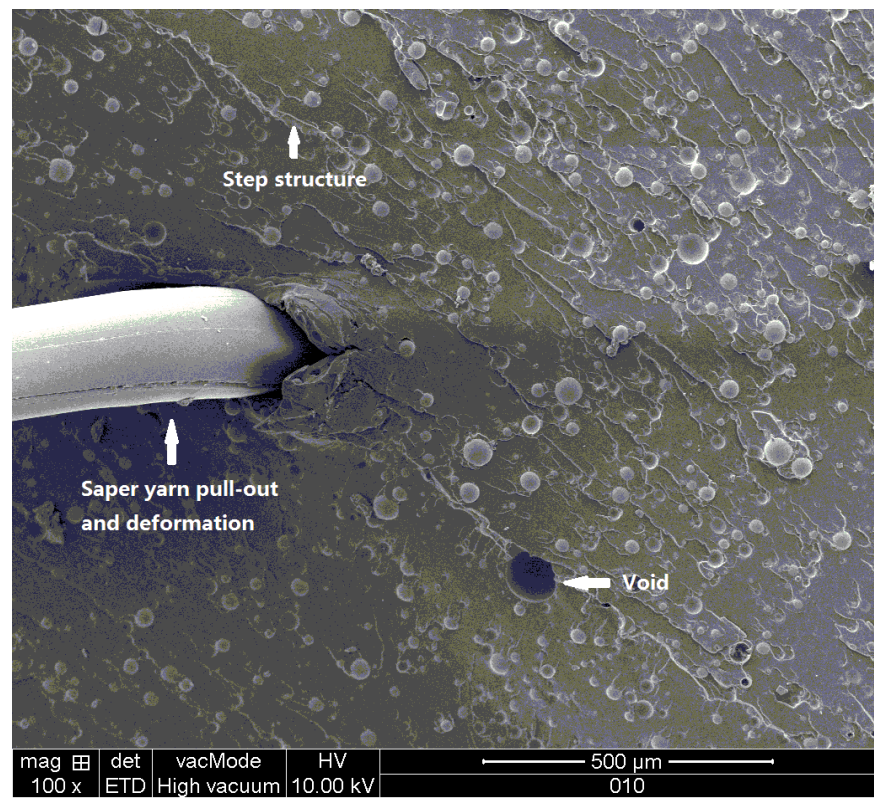

Figure 10. SEM micrograph of the fracture surface of S1 after TPB tests

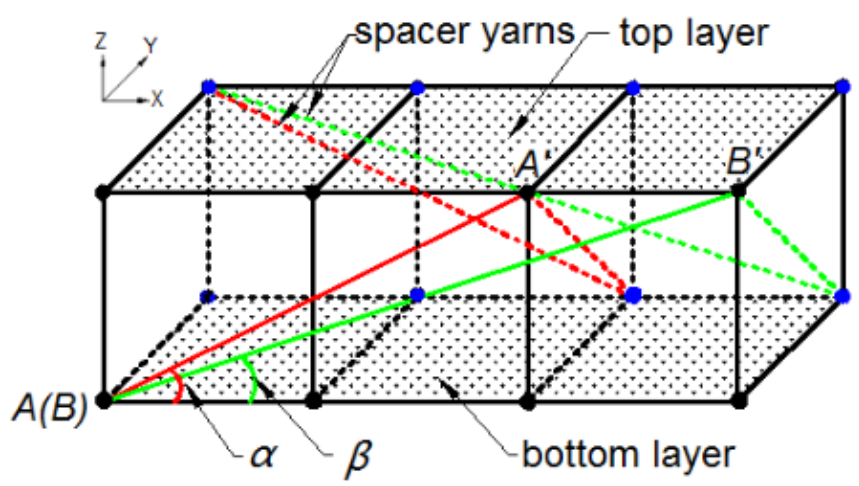

Figure 11. The global lapping movements of spacer yarns carried by GB3

to a better structural integrity, stronger surface layer and more spacer yarns in per unit area, resulting in an obvious increase of the bending responses of $\mathrm{S} 1$.

\subsection{Effects of microballoons on flexural properties}

As one of the components, S60HS microballoons with content $30 \%, 20 \%$ and $40 \%$ were used to fabricate specimens S1, S5 and S6, respectively. The flexural strength, specific flexural strength, modulus and specific modulus values of these three samples are shown in Figures 12 and 13, respectively. It can be seen that $\mathrm{S} 5$ exhibits the lowest flexural strength and flexural modulus values, while flexural strength and flexural modulus values of S1 and S6 are very close and higher than that of S5. Additionally, the lower density of microballoons and more voids generated during the manufacturing process increase the volume fraction of microballoons, leading to the decrease of density of the whole composite (Table 4). Hence, in the case of specific properties of SF-WKSF, S6 has relatively higher values compared with $\mathrm{S} 1$ and $\mathrm{S} 5$. In summary, when the volume content of $\mathrm{S} 60 \mathrm{HS}$ microballoons is relatively low, the bending performance of SF-WKSF can be 
significantly improved by increasing the volume fraction of $\mathrm{S} 60 \mathrm{HS}$ microballoons. However, when the content of S60HS microballoons reached a certain level, the specimen with higher volume fraction shows superior specific flexural mechanical properties, but the effects of content on flexural strength and modulus are not significant. The high-compressive-strength S60HS microballoons distributed and embedded in the matrix resin increases the quantity of step structures when the volume fraction of S60HS content is low, resulting in the increase of anti-bending capacities of specimens. Further increasing the volume fraction of microballoons can increase the number of voids and agglomeration between microballoons, which slow down the increasing of flexural strength and modulus of SFWKSF.

Specimens S1 and S7 were filled with the different type of microballoons (S60HS and $\mathrm{K} 1$, respectively). The flexural strength, specific flexural strength and modulus, specific modulus values of S1 and S7 are shown in Figure 14 and 15, respectively. It is evident that the sequences of all these items of composites are S1>S7, which means that the flexural properties of SF-WKSF increase with the increase of microballoons density (Table 1). Figure 16 and 17 show the SEM micrographs of the fracture surface of S7 after TPB tests with low and high magnification, respectively. Similar to the SEM micrograph of S1 (Figure 10), the microballons fracture and debonding can also be found in S7. Differently, the spacer yarns are not pulled out and deformed in S7, but some crushed microballons were found and distributed in the whole matrix resin. In addition, the large area of microballoons fracture can be found in the high magnification SEM image, as shown in Figure 17. Hence, the $\mathrm{K} 1$ microballoons are one of the primary load carriers of SFWKSF during the TPB tests. The phenomena are resulting from the low strength of $\mathrm{K} 1$ microballoons. According to the least resistance path theory, K1 microballoons cannot resist and change the propagation direction of crack, this indicates that the less step structures can be generated as a result of less consumption of flexural energy during TPB tests. All the results degrade the flexural performance of S7.

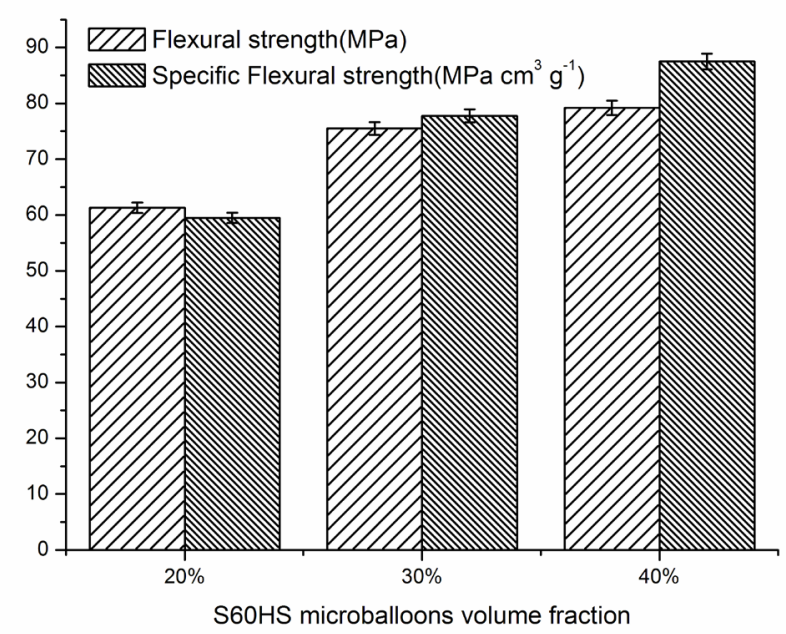

Figure 12. The flexural strength and specific flexural strength of specimens with different microballoon volume fraction

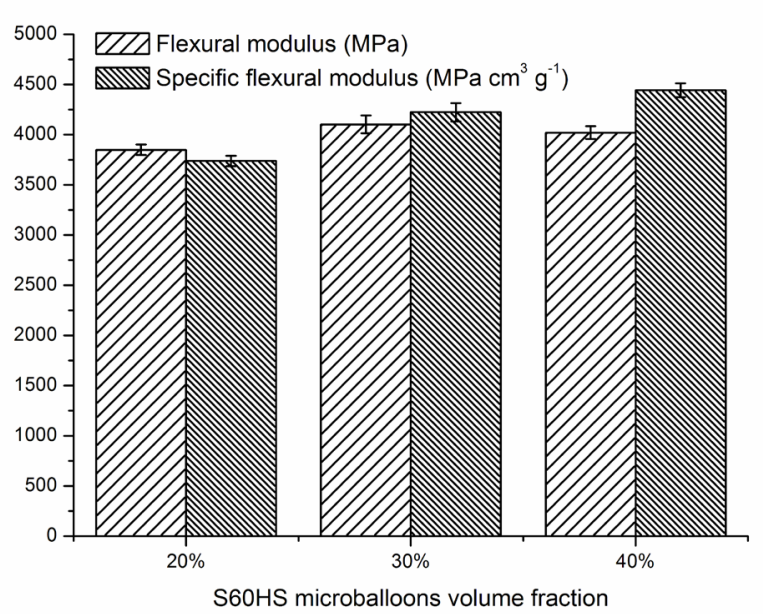

Figure 13. The flexural modulus and specific flexural modulus of specimens with different microballoon volume fraction

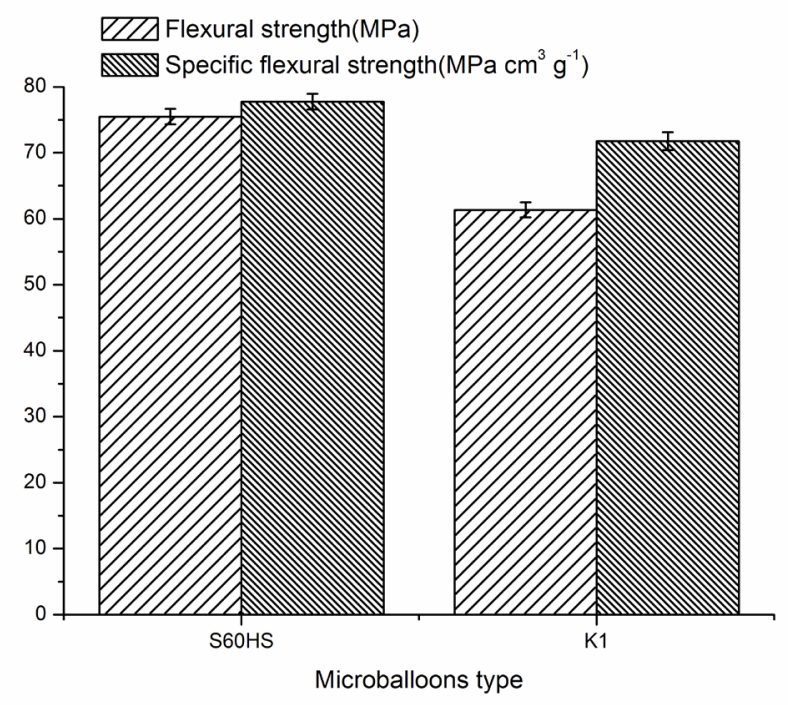

Figure 14. The flexural modulus and specific flexural modulus of specimens with different microballoon type

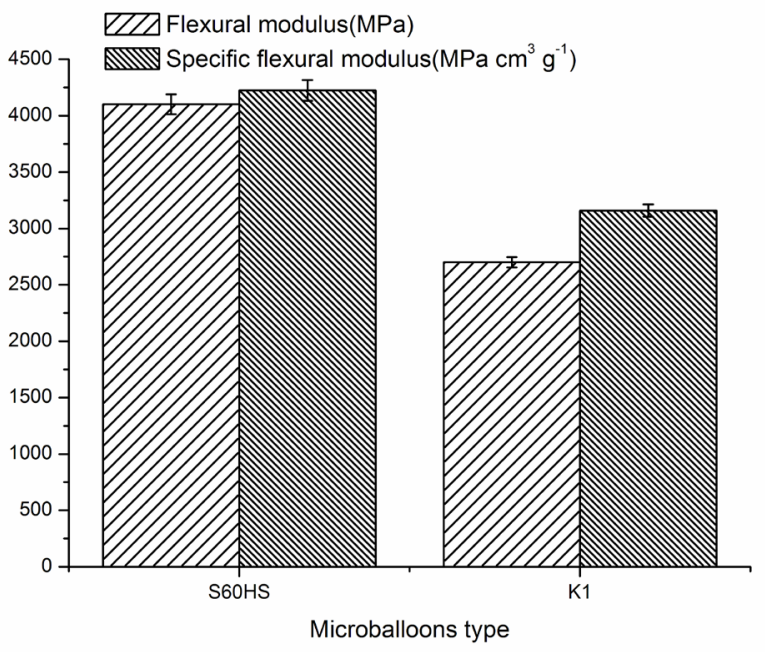

Figure 15. The flexural modulus and specific flexural modulus of specimens with different microballoon type 


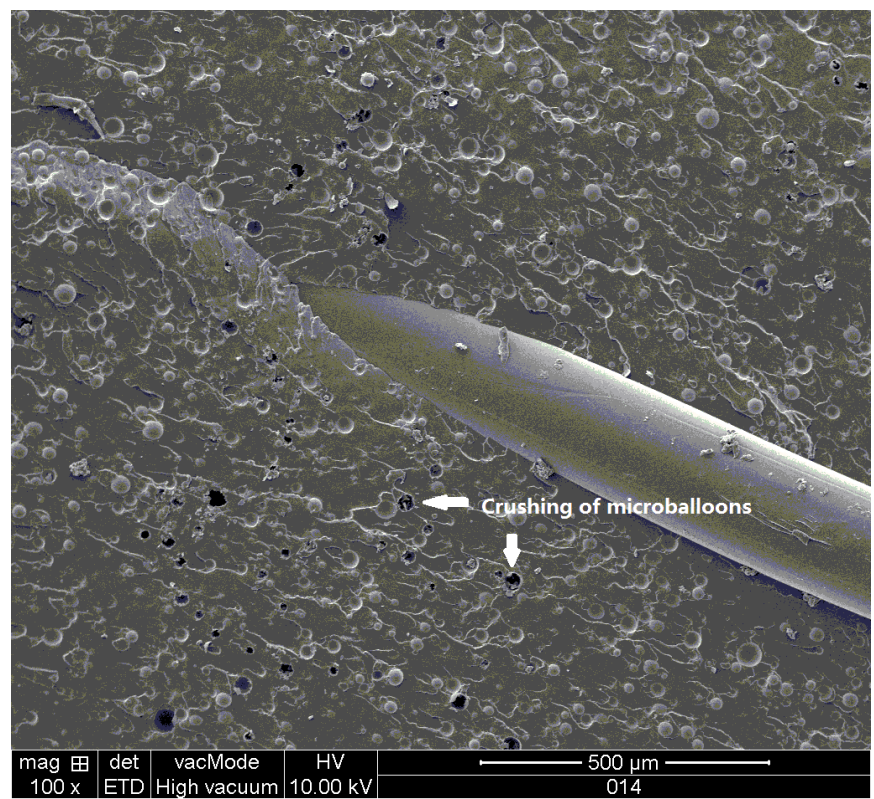

Figure 16. The SEM micrograph of the fracture surface of S7 after TPB tests (low magnification)

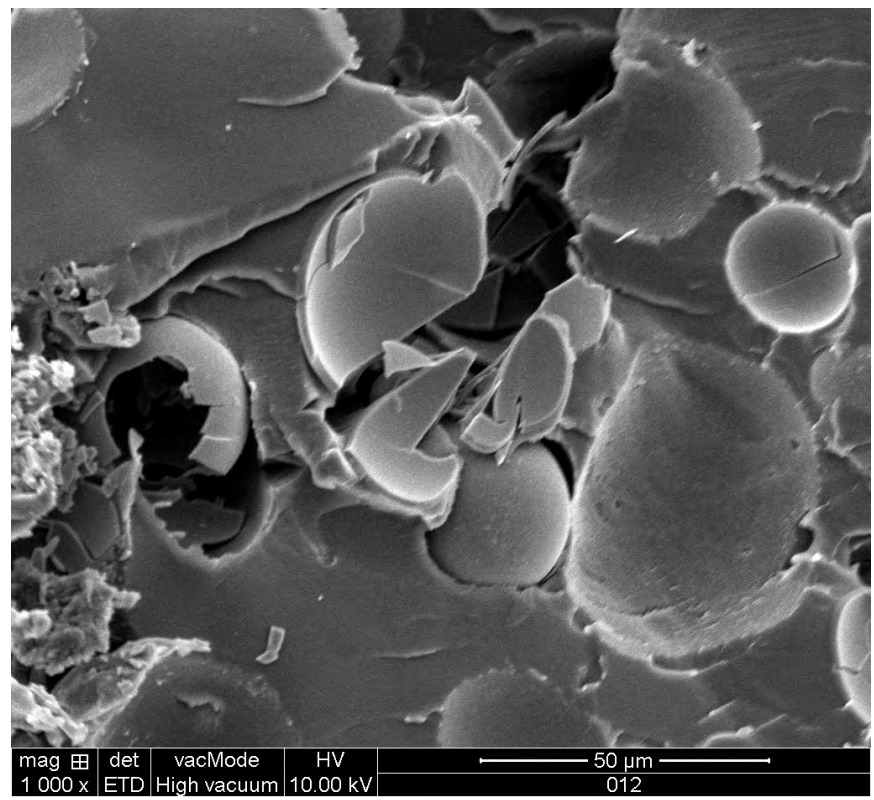

Figure 17. The SEM micrograph of the fracture surface of S7 after TPB tests (high magnification)

\section{Conclusions}

In this paper, a new type of composite concerning SF-WKSF was prepared and the TPB tests were carried out to investigate the flexural properties of this material. Based on the experiment results, the effects of WKSF and microballoons on flexural strength and modulus of this composite were analysed.

The results showed that the SF-WKSF displays superior properties than NSF with respect to the flexural strength and modulus.

The bending properties of SF-WKSF were obviously controlled by some structural parameters of WKSF. The composites with larger inclination-angle and closer surface layer structure exhibit better performance on the flexural capacities. However, the effect of fineness of the spacer yarns on anti-bending capacities is not significant. Therefore, it is important to select the appropriate fabric structural parameters during the production of this material.

Different types and content of microballoons have obvious influence on the bending responses of specimens, which can be improved by using microballoons of higher density and increasing content of $\mathrm{S} 6 \mathrm{OHS}$ microballoons within an appropriate range.

The research introduces valuable technical information for fabrication of this composite and application as aero, marine and construction materials.

\section{ACKNOWLEDGEMENTS}

The authors thank the financial supported by "the Fundamental Research Funds for the Central Universities" [grant number 14D310104].

\section{References}

[1] Karthikeyan, C. S., and Sankaran, S. (2000). Influence of chopped strand fibres on the flexural behaviour of a syntactic foam core system. Polymer international, 49(2), 158-162.

[2] Hu, G., Yu, D. (2011). Tensile, thermal and dynamic mechanical properties of hollow polymer particle-filled epoxy syntactic foam. Materials Science and Engineering: A, 528(15), 5177-5183.

[3] Liang, J. Z. (2005). Tensile and flexural properties of hollow glass bead-filled ABS composites. Journal of elastomers and plastics, 37(4), 361-370.

[4] Rizzi, E., Papa, E., Corigliano, A. (2000). Mechanical behavior of a syntactic foam: experiments and modeling. International Journal of Solids and Structures, 37(40), 5773-5794.

[5] Papa, E., Corigliano, A., Rizzi, E. (2001). Mechanical behaviour of a syntactic foam/glass fibre composite sandwich: experimental results. Structural Engineering and Mechanics, 12(2), 169-188.

[6] Gupta, N., Woldesenbet, E. (2005). Characterization of flexural properties of syntactic foam core sandwich composites and effect of density variation. Journal of composite materials, 39(24), 2197-2212.

[7] Karthikeyan, C. S., Sankaran, S. (2005). Flexural Behaviour of Fibre-Reinforced Syntactic Foams. Macromolecular Materials and Engineering,290(1), 60-65.

[8] Ferreira, J. A. M., Capela, C., Costa, J. D. (2010). A study of the mechanical behaviour on fibre reinforced hollow microspheres hybrid composites. Composites Part A: Applied Science and Manufacturing, 41(3), 345-352.

[9] Zhang, L., Ma, J. (2013). Effect of carbon nanofiber reinforcement on mechanical properties of syntactic foam. Materials Science and Engineering: A, 574, 191-196.

[10] Wang, L., Zhang, J., Yang, X., Zhang, C., Gong, W., $Y u$, J. (2014). Flexural properties of epoxy syntactic foams reinforced by fiberglass mesh and/or short glass fiber. Materials \& Design, 55, 929-936. 
[11] Guo, X., Long, H., Zhao, L. (2013). Investigation on the impact and compression-after-impact properties of warpknitted spacer fabrics. Textile Research Journal, 83(9), 904-916.

[12] Liu, Y., Hu, H., Zhao, L., Long, H. (2011). Compression behavior of warp-knitted spacer fabrics for cushioning applications. Textile Research Journal, 82(1), 11-20.

[13] Velosa, J. C., Rana, S., Fangueiro, R., Van Hattum, F. W. J., Soutinho, F., Marques, S. (2012). Mechanical behavior of novel sandwich composite panels based on 3D-knitted spacer fabrics. Journal of Reinforced Plastics and Composites, 31(2), 95-105.
[14] Chen, S., Long, H. R. (2014). Investigation on compression properties of polyurethane-based warp-knitted spacer fabric composites for cushioning applications Part $I$. Experiment. INDUSTRIA TEXTILA, 65(4), 200-205.

[15] Maharsia, R., Gupta, N., Jerro, H. D. (2006). Investigation of flexural strength properties of rubber and nanoclay reinforced hybrid syntactic foams. Materials Science and Engineering: A, 417(1), 249-258.

[16] Gupta, N., Woldesenbet, E. (2005). Characterization of flexural properties of syntactic foam core sandwich composites and effect of density variation. Journal of composite materials, 39(24), 2197-2212. 